\title{
Design and Implementation of Microcontroller based Autonomous Wireless Sensor Network for Predicting and Forecasting of Fire Accident in a Multi-Store Building
}

\author{
Mehari Kiros \\ PhD scholar, \\ Andhra University College of Engineering (A), \\ Andhra Pradesh, Visakhapatnam-530003, India
}

\author{
S. Pallam Setty, PhD \\ Professor \\ Andhra University College of Engineering (A), \\ Andhra Pradesh, Visakhapatnam-530003, India
}

\begin{abstract}
Safety is the most important issue to rescue the lives of people and damage of assets. To achieve this goal, a fire accident existence predictor which also avoids hazard is crucial. The system is sensitive to temperature and pressure above the threshold values thereby produces different kinds of signals to stimulate that a fire may take place in the multi-store building at some time and location. In this paper, it has been developed a prototype of an autonomous wireless sensor network predicting and forecasting fire accident in a multi-store building. This prototype is a low cost, efficient and portable. It also is capable of avoiding the fire accident. The system displays when and where the event takes place and gives alarm signals in the form of visual, audible and SMS on LEDs and LCD, buzzer and user mobile phones respectively.
\end{abstract}

\section{General Terms}

GSM Network; Autonomous; LCD and LED displays; hazard indicators; signals.

\section{Keywords}

Predicting; Forecasting; wireless Sensor Network; Potential fire.

\section{INTRODUCTION}

Nowadays, global warming is a burning issue throughout the world. Climate change is a result of changes in the physical world caused either due to natural or human factors and can lead to disaster. The goal is to come out with a device which serves as fire accident predicting and forecasting, detecting and avoiding system. When there is a situation that a fire is going to take place, the system generates different hazard indicator signals such as the buzzer, LCD, LEDs displays and SMS to user's cell phones using GSM Network and send signal to the servo motor controller to open the fire extinguisher hose in case of fire accident. The system which senses the fire potential also transmits an SMS to the other fire detecting systems (nodes) in the network, so that these nodes, in turn, will send signal to the respective buzzers, LCD, LEDs and send SMS to users' mobile phone.

\subsection{Wireless Sensor Network}

A wireless Sensor Network is the liking of two or more electronics devices that utilize the frequency modulation technique based on radio waves without physical connection in the local area network. As the name portrays, there is no physical connection among the electronic devices, the communication is then achieved through the radio waves.
There is a high mobility in wireless communication within the limited coverage area and remains connected.

\subsection{Sensing and Sensors}

Sensing is reading some kind of physical world parameters. For instance, a temperature and pressure sensors; it is through these sensors that the system can collect the information of the surrounding which helps it to control the variation in pressure and temperature; taking different kinds of actions when there is something lead to catastrophic. A sensor then is a device which senses physical environmental parameters and produces an electrical signal at its output terminal in either analog or digital form depending on the type of the sensor used.

In a narrow sense, a sensor is a device which transforms physical parameter into electrical signals. Subsequently, the resulting signals may need further processing for best matching of the next device which takes these signals as input. Some of the signal processing techniques are signal amplification, to get the sensor output signal to bring up to the required level to best match with the next device input level. Voltage level shifting, for example, when there is a need to communicate through the RS232 standard Serial ports. Analog to digital conversion (ADC) or the other way round digital to analog conversion (DAC). The sensor output signals may also need to step down if the matching device next to the sensor supports low level signal, this time attenuation technique comes into picture. The next job is to use the wellconditioned signal for actuation as shown in Fig.1 it can be seen that the common data acquisition and actuation block diagram is also used in sensor data acquisition and actuation techniques.

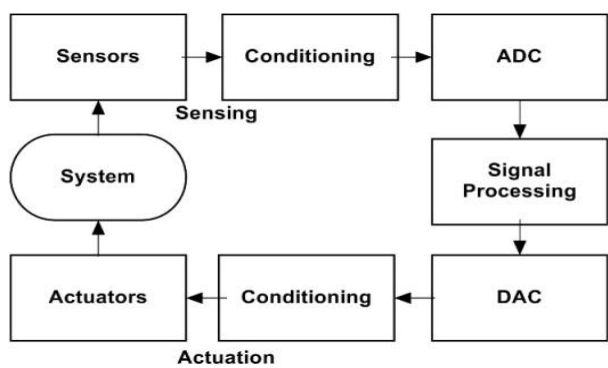

Fig.1. Data acquisition and actuation [7]

\section{RELATED WORK}

Today, Smart devices are highly affecting the way we live. Like the smart home, house appliances wirelessly controlled kitchen and so on are contributed by the wireless sensor networks. Wireless Sensor Networks are also applied in many 
other sectors such as the Telecommunication sector, environmental monitoring sector, and healthcare sector agricultural sector and in industrial applications. Moreover, they are applied in man's daily activities to make the habitat easy and smarter than ever before. Examples of the above application areas are fire station, robotic-surgery, and smart plant monitoring and product inspection in manufacturing industry and the like ones. While surveying, what is done yet related to this topic is, many authors come out with different applications in the area of wireless sensor network using different approaches depending on their desired goals. These related works are covered in general speaking. this system is Microcontroller Based Autonomous Wireless Sensor Network for Predicting and Forecasting of Fire accident in a MultiStore building developed with the help of Proteus and MikroC Software. The system has been tested in different scenarios for its robustness and reliability. it displays when and where the event takes place on mobile phones as well as on LEDs and LCD within a delay of $300 \mathrm{~ms}$ time and gives alarms in the form of visual, audible and SMS on LEDs and LCD, buzzer and user mobile phones respectively. In fact, the delay time depends on many factors, the system is emulated on core i5 system and software, coded on MikroC and Circuit Emulator is proteus.

\section{EXPERIMENTAL SETUP \\ 3.1 The GSM module}

The Global System for Mobile (GSM), irrespective of the advancements of the information communication technologies, GSM has is still the dominating kind of communication technology leading and abundantly used throughout the world and paved a new way for mobile communication [5]. In the design, interfaced GSM Module is interfaced with a PIC microcontroller through the RS232 standard serial port. When there is detection of fire potential in a floor, the GSM module will send SMS to the user by initiating that fire has been detected and it also displays the time and location of the hazard occurred on LCDs in addition to the hazard indicators and alarms. As a result a fire extinguisher mechanism integrated with a servo motor is armed accordingly.

When a potential fire is detected different kinds of Signals are generated by the system; the transmitter sends SMS a fire is to take place in a particular floor of a Multi-Store building to mobile phones of users and to other nodes in the network to alert them to set on the audible, visible alarms and SMS the time and location of the fire accident to cell phones. The receiver is the other terminal located in the network nodes and user's mobile; receives the signal from the transmitters of the fire detecting node(s) and relays that signal to the microcontroller for further processing and generation of fire avoiding mechanism to the servo motor controller and alarms for evacuating people around the fire zone.

\section{THE PROCESSING UNIT}

Two microcontrollers are used incostruction of the prototype, one is receiving the sensor values, computing the analog inputs, and generate corresponding signals based on the sensor values to the LEDs, buzzer, to the Servo Motor controller which opens the fire extinguisher hose in case of fire and to the timing event generator microcontroller so that it will initiate the SMS to user mobile phone at which a fire accident is ging to take place in a particular floor of the MultiStore building. There are also displays that the current values of the sensors on LCD. The second microcontroller generates a real time of events taking place, displays the date and time of events and sends a signal to the GSM module. These microcontrollers are the hearts of the system. Here the RTC (real time clock) IC is used to generate the timing events at which a fire accident has occurred. Then SMS message is updated through the RS232 standard serial port using the HyperTerminal as a receiver in the simulation.

\subsection{Power Supply}

A Power Supply is the most mostly important issue in the areas of Wireless sensor networks. Since most electronics devices lifetime and reliability is mostly dependent on the energy consumed by the device. This system needs also regulated power supply for proper functioning and for long life of the system. In wireless sensor nodes power sources are batteries in most cases which can be rechargeable or disposable kinds of DC sourcing batteries.

\section{THE SOFTWARE}

Why Proteus and MikroC? The reason is, Proteus VSM for PIC contains everything required to develop; test and virtually prototype a system designs based around the Microchip Technologies ${ }^{\mathrm{TM}}$ PIC16 series of Microcontrollers. The unique nature of Proteus is schematic based Microcontroller simulation which facilitates rapid, flexible and parallel development of both the system hardware and the system firmware. This design synergy allows engineers to evolve their projects works more quickly, rapid prototyping, empowering them with the flexibility to make hardware or firmware changes and will reduce the time to market $[5,14]$. The MikroC for PIC programming environment is used to develop the firmware which looks like a $\mathrm{C}$ programming language that is efficient and user friendly for those who experienced $\mathrm{C}$ language.

\section{THE HARDWARE DESIGN}

The design and implementation of the fire accident predicting and forecasting system is accomplished by taking two analog inputs of pressure and temperature sensors computing the input and deciding to generate corresponding signals based on the results of both inputs or either of them using the OR gate logic. To develop the prototype of the system, we use PIC Microcontrollers as processing units. This has been developed the complete MikroC Source Code and Proteus Schematic design of the prototype system. The continuous Signal (analog) output voltage of pressure and temperature sensors is given to the analog Input pins of the PIC Microcontroller. The built in ADC in PIC16 series Microcontrollers is 10-bit. The result of the 10-bit Analog to Digital Converter (ADC) is read using the function ADC Read. This 10-bit digital value is then converted to the corresponding voltage levels and ready for manipulations and formatting depending on the type of output we desire to display. For instance in this case LCD display is used, therefore the signal should be in an appropriate format and then get displayed.

\subsection{The ADC Module of Pic Microcontroller}

Analog to Digital Converter (ADC) is a device that converts a continuous voltage signal to discrete digital values. To deal with some processing on physical quantities, which are normally continuous signals in nature, ADC plays a key role. ADC module is integrated in most PIC Microcontrollers. Here, PIC16 series Microcontrollers are used for the prototype development. The ADC Converter module can also operate in sleep mode in which clock is derived from its internal RC oscillator. 


\subsection{Quantization Error}

When the ADC input is -Vref, result will be 0000000000 and When the ADC input is +Vref, result will be 1111111111(binary),

Threshold of ADC $=(+$ Vref $--V r e f) /(210-1)$, is one of the counts out of the 1024 steps

Here ADC threshold $=(5-0) /(1024-1)=5 / 1023=$ $0.004887 \mathrm{~V}$ So, if the input is $5 \mathrm{~V}$, ADC Value is $5 / 0.004887=$ $1023=11111111$ (binary). If the input is $0.004887 \mathrm{~V}, \mathrm{ADC}$ Value is $0.004887 / 0.004887=1$.

\subsection{The Temperature and Pressure Sensors}

In recent times, temperature sensors come in different varieties in their calibration, digital or analog type, range of operation and in IC form or in a kind of wires. The temperature sensor used is (LM35) in the prototype development as it is found low cost, easily available in market as well as in simulators, emulators and above all it is found that suitable for the prototype device. This sensor is an analog sensor produced by National semiconductor calibrated in centigrade scale and its operation range is $-55 \mathrm{oC}$ to $150 \mathrm{oC}$. It is also used in military equipment due to its high range of operation. The output of the sensor changes $10 \mathrm{mV} / 1^{\circ} \mathrm{C}$. The pressure sensor (MPX4115) is also used in the system to sense the change in surrounding pressure. This sensor is an analog pressure sensor too, which produces analog signal output on its output terminal. The internal circuitry of this sensor is detailed in the datasheet and can be referred for further explanation and understanding the graphically explained transfer function of this sensor.

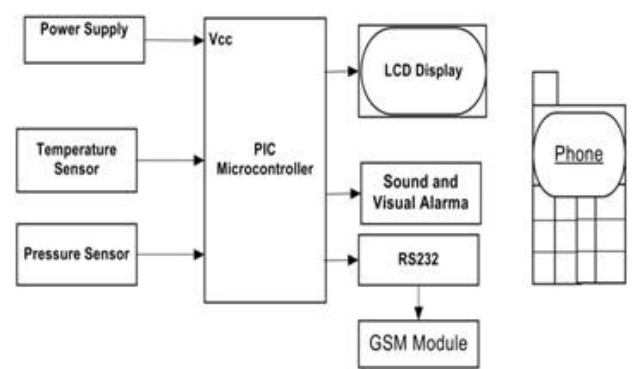

Fig.2. the system block diagram
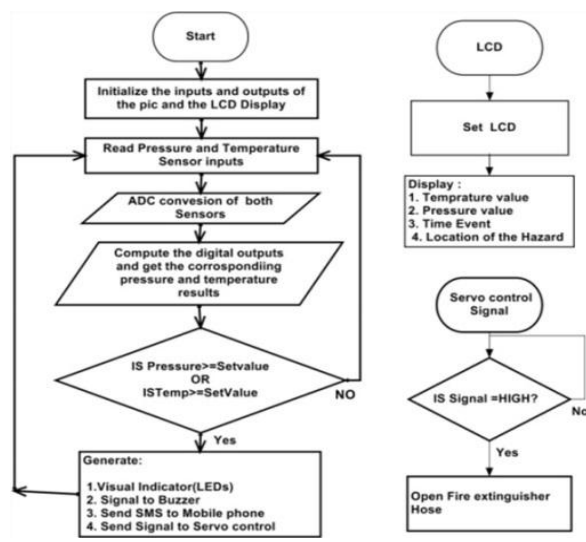

Fig.3. the system flow charts

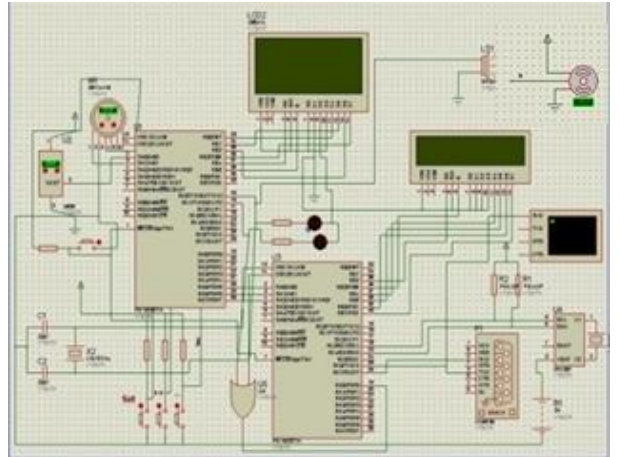

Fig.4.the system Circuit Diagram before simulation

\section{SIMULATION RESULTS IN DIFFERENT SCENARIOS}

1. The system displays in which floor a potential fire is going to take place on LCD.

2. The system indicates a potential fire through different signals such as alarm buzzer, visual LEDs and SMS messages within $300 \mathrm{~ms}$ time.

3. The system generates a signal to servo motor control to open the fire extinguisher hose to avoid hazard.

4. The system automatically stops all the signaling when the hazard is avoided, which means we can see the time taken to avoid the disaster.

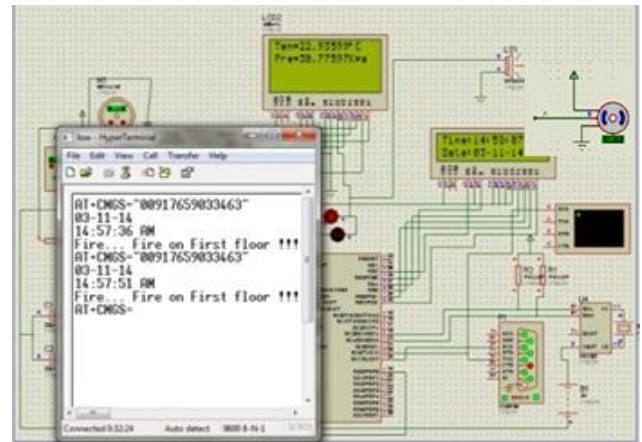

Fig.5. System response when temperature and pressures are below threshold value

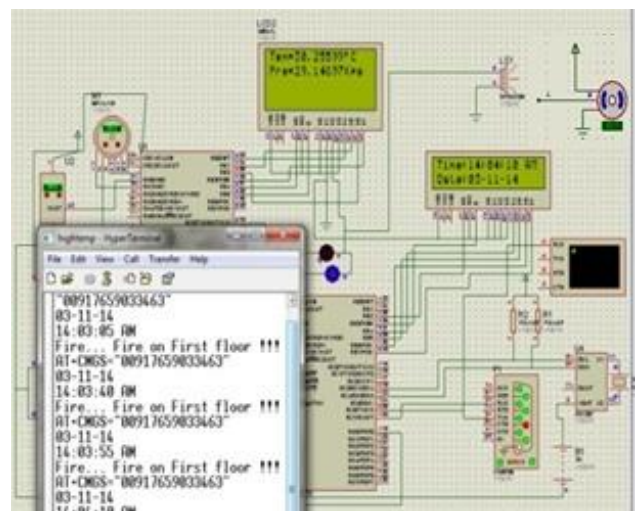

Fig.6. System response at high pressure 


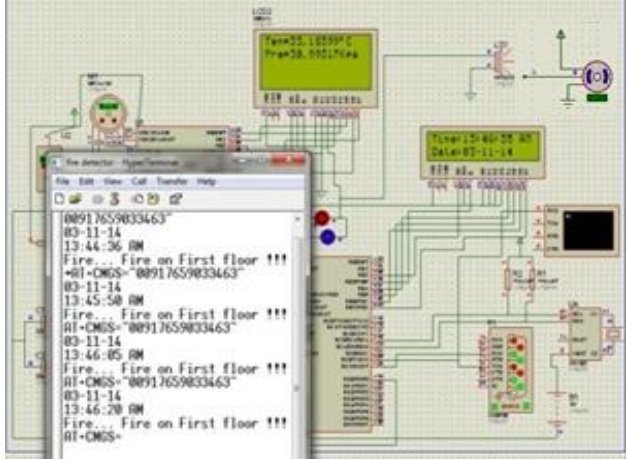

Fig.7. System response at high temperature

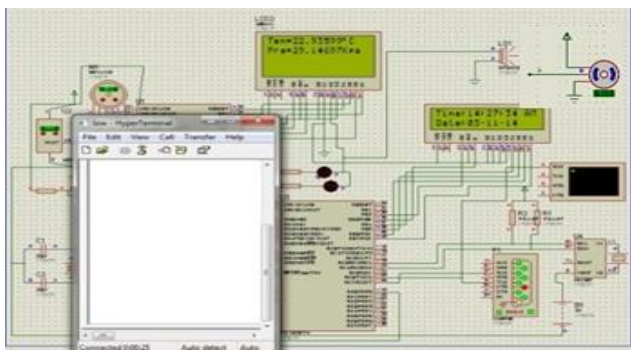

Fig.8. System response at high pressure and temperature

\section{CONCLUSIONS}

The developed system is a Microcontroller based prototype of an Autonomous Wireless Sensor Network for fire accident Predicting and forecasting in Multi-Store Building. The system is very accurate while responding in a time of $300 \mathrm{~ms}$. The system has been tested in different scenarios for its robustness and reliability. This system displays when and where the event takes place within a delay of $300 \mathrm{~ms}$ time and gives alarms in the form of visual, audible and SMS on LEDs and $\mathrm{LCD}$, buzzer and user mobile phones respectively. In fact, delay time depends on many factors; the system is emulated on core i5 system and software, coded on MikroC and Circuit Emulator is proteus.

\section{ACKNOWLEDGMENTS}

I would like to thank to the Ethiopian Ministry of Defence and Andhra university college of Engineering, Department of Computer Science and Systems Engineering for supporting me to do my research work.

\section{REFERENCES}

[1] J. Bennet Solomi*, M. Rajalakshmi**,C.V. Mala**, December 2012,GPRS Based HealthCare Telemonitoring System, International Journal of Scientific and Research Publications, Volume 2, Issue 12, ISSN 2250-3153

[2] Iman Morsi1,a and Loay Mohy El-Din Rasheed, 2013, Microcontroller System for Oil Refinery Parameters Measurements Based on Piezoresistive and Strain Gauge Pressure Sensors, Applied Mechanics and Materials Vols. 249-250, pp 1133-1138, Trans Tech Publications,
Switzerland

doi:10.4028/www.scientific.net/AMM.249-250.1133

[3] N. Anju Latha1*, B. Rama Murthy1, U. Sunitha2, September- October 2012, Design and Development of A Microcontroller Based System for the Measurement Of Blood Glucose, International Journal of Engineering Research and Applications (IJERA) ISSN: 2248-9622, Vol. 2, Issue 5.

[4] Rashedul Qayum nimA lunashE M.S Md Kamrul Hussain, October-2012, Enhanced Wireless Control System for Smoke and Fire Detection, International Journal of Scientific and Engineering Research, Volume 3, Issue 10,ISSN-2229-5518.

[5] Sunil MP, Ashik Narayan, Vidyasagar Bhat, Vinay S, August 2013, Smart Biogas Plant, International Journal of Innovative Technology and Exploring Engineering (IJITEE) ISSN: 2278-3075, Volume-3, Issue-3.

[6] Manaswi M. Latthe1, S. B. Patil, February 2014, Online Monitoring and Alarm Indication of Status of Air for Automation, International Journal of Science and Research (IJSR) ISSN (Online): 2319 7064,Volume3,Issue-2.

[7] Fundamentals of Wireless Sensor Network Theory and Practice, 2010, A John Wiley and Sons, Ltd., Publication.

[8] F. van den Bergh ,G. Udahemuka, B. J. vanWyk,2009,Potential Fire Detection Based on Kalman-Driven Change Detection, IGRASS.

[9] Maria Skrinska,Jan Skrinsky, Petr Dolnicek,Petra Lukesova,

Jan Marek, 2014, Flash-Point Prediction for Industrial Applications, International Conference on Mathematics and Computers in Sciences and in Industry.

[10] N. Anju Latha and B. Rama Murthy,CH.V.V.Ramana and

Willem Clarke, K. Venu Madhav , V.Siva Kuma,2011, Design and Development of A Microcontroller Based System for the Measurement of Blood Pressure, International Conference on Recent Advancements in Electrical, Electronics and Control Engineering.

[11] Kerstin Wendt, Ana Cortes, Toma's Margalef, 2010, Evolutionary Intelligent System for Input Parameter Optimization in Environmental Modelling: A Case Study in Forest Fire Forecasting.

[12] MilyJashank, Sureshchandra J Gupta, Jayalakshmi M Nair, 2014, Analysis of Environmental Temperature Variation as a Precursor of Earthquake Prediction Using I2C,Annual IEEE India Conference (INDICON)

[13] Data sheets of pic16f877A, LM35 and MPX4115

[14] www.labcenter.co.uk 